\title{
More promising results of liver intravoxel incoherent motion imaging analysis for the assessment of nonalcoholic steatohepatitis and fibrosis
}

\author{
Yì Xiáng J. Wáng ${ }^{1 \wedge}$, Ben-Heng Xiao ${ }^{1 \wedge}$, Cun-Jing Zheng ${ }^{1}$, Ting Li $^{2}$, Nazmi Che-Nordin ${ }^{3}$, Wei Wang ${ }^{2}$ \\ ${ }^{1}$ Department of Imaging and Interventional Radiology, Faculty of Medicine, The Chinese University of Hong Kong, Shatin, New Territories, \\ Hong Kong SAR, China; ${ }^{2}$ Department of Radiology, The Third Xiangya Hospital, Central South University, Changsha, China; ${ }^{3}$ College of Health \\ Sciences, Gulf Medical University, Ajman, United Arab Emirates \\ Correspondence to: Dr. Yì Xiáng J. Wáng. Department of Imaging and Interventional Radiology, Faculty of Medicine, The Chinese University of \\ Hong Kong, Shatin, New Territories, Hong Kong SAR, China. Email: yixiang_wang@cuhk.edu.hk. \\ Comment on: Troelstra MA, Witjes JJ, van Dijk AM, et al. Assessment of Imaging Modalities Against Liver Biopsy in Nonalcoholic Fatty Liver \\ Disease: The Amsterdam NAFLD-NASH Cohort. J Magn Reson Imaging 2021. [Epub ahead of print]. doi: 10.1002/jmri.27703.
}

Submitted Jul 02, 2021. Accepted for publication Jul 26, 2021.

doi: $10.21037 /$ atm-21-3442

View this article at: https://dx.doi.org/10.21037/atm-21-3442

Diffusion-weighted imaging (DWI) has many advantages. It is widely available among MRI scanners, and has the ability to provide both tissue diffusion and perfusion information without an intravenous contrast agent or additional hardware. Le Bihan et al. (1) proposed the intravoxel incoherent motion (IVIM) principle of DWI, which provides the estimation of quantitative parameters reflecting tissue diffusivity [slow diffusion $\left(\mathrm{D}_{\text {slow }}\right)$ ] and tissue microcapillary perfusion [PF (perfusion fraction) and fast diffusion $\left.\left(D_{\text {fast }}\right)\right]$. We proposed that the relationship between liver DWI signal and $b$-values can be separated into two parts: part- 1 is the signal difference between the $b=0 \mathrm{~s} / \mathrm{mm}^{2}$ image and the first very low $b$-value image; the rest is part2 and fitted with a bi-exponential decay model starting from the first non-zero very low $b$-value data $(2,3)$. On DWI, blood vessels show a high signal when there is no diffusion gradient $\left(b=0 \mathrm{~s} / \mathrm{mm}^{2}\right)$ and a low signal even when very low diffusion gradient values (e.g., $b=1 \mathrm{~s} / \mathrm{mm}^{2}$ ) are applied. Therefore, for part- 1 , the signal difference between images when the diffusion gradient is off and images when the diffusion gradient is on, reflects the extent of tissue vessel density [referred to as diffusion-derived vessel density (DDVD)] $(2,4)$. For part-2, starting from a nonzero very low $b$-value, the relationship between DWI signal and $b$-value follows a bi-exponential decay $(2,3)$. It has been shown that DDVD is correlated with the IVIM parameters and is a useful biomarker for the separation of livers with and without fibrosis, and livers with severe fibrosis tend to have even lower DDVD measurement than those with milder liver fibrosis (4). Recently, we demonstrated that liver parenchyma in healthy subjects has an agingdependent decrease of DDVD and $\mathrm{D}_{\text {slow }}(5,6)$. As the magnitude of aging-related liver diffusion metrics changes is expected to be small compared with pathology induced changes, our results suggest the relative precision of liver diffusion metrics quantification.

We also published results which strongly support the clinical usefulness of liver IVIM analysis. The report of Wáng et al. (7) had 16 healthy volunteers and 33 hepatitis B liver fibrosis patients, among them 15 patients had stage 1 liver fibrosis; the report of Huang et al. (8) had 26 healthy volunteers and 12 hepatitis B liver fibrosis patients, among them four patients had stage 1 liver fibrosis; the report of Li et al. (9) had 20 healthy volunteers and 28 hepatitis B liver fibrosis patients, among them 11 patients had stage 1 liver fibrosis. All patients and healthy volunteers in these

^ ORCID: Yì Xiáng J. Wáng, 0000-0001-5697-0717; Ben-Heng Xiao, 0000-0003-1575-1475. 
three studies can be separated by IVIM analysis, except for one case of stage 2 fibrosis in the study of $\mathrm{Li}$ et al (9). Interestingly, the studies of Huang et al. and $\mathrm{Li}$ et al. each had four patients with biopsies showing no fibrosis, and these eight subjects' diffusing MRI measurements resembled those of healthy volunteers $(8,9)$. Moreover, we recently completed an animal-based study using the biliary duct ligation (BDL) model (10). Liver diffusion MRI was conducted with male Sprague-Dawley rats (weight: $300 \pm 20 \mathrm{~g})$, and included control rats $(\mathrm{n}=6)$, and experimental rats of 7 days $(\mathrm{n}=5), 14$ days $(\mathrm{n}=5), 21$ days $(\mathrm{n}=5)$ and 28 days $(\mathrm{n}=3)$ after BDL surgery. In addition, 12 rats had BDL for 7 days and then under partial biliary duct recanalization status for a mean of additional 14 days. Of the rats with surgery, 4, 12, 7, and 6 rats had stage 1, 2, 3, 4 liver fibrosis respectively (one BDL day-21 rat missed histology). Our results show IVIM and DDVD analysis allowed total separation of healthy rat livers and fibrotic rat livers (10).

While our liver diffusion MRI was conducted with three clinical studies on liver fibrosis due to viral hepatitis B and one animal study with biliary blockage induced liver fibrosis, recently Troelstra et al. (11) reported a liver diffusion MRI study on nonalcoholic fatty liver disease (NAFLD) with very promising results. The Amsterdam NAFLD-NASH cohort (ANCHOR) study is an observational prospective study aiming to identify and validate noninvasive diagnostic methods, both imaging and molecular markers, for the assessment of NAFLD disease spectrum. The first 37 individuals ( 23 men, 14 women) with hepatic steatosis on ultrasonograph were included in this report. The imaging modalities included ultrasonographic elastography, MR elastography (MRE), IVIM, and three MR approaches to quantify liver fat (MR spectroscopy, magnitude-based MRI proton density fat fraction, and three-point Dixon). MRI was conducted at 3.T scanner. MRE was performed using a gravitational transducer at $50 \mathrm{~Hz}$ and a generalized multishot gradient-recalled echo. Stiffness based on the shear modulus (G') and loss modulus (G') were determined. The IVIM sequence consisted of a free breathing multislice diffusion weighted single-shot echo-planar imaging sequence with $18 b$-values $(0,1,2,5,10,20,30,40,50,75$, $\left.100,150,200,300,400,500,600,700 \mathrm{~s} / \mathrm{mm}^{2}\right)$. PF, $\mathrm{D}_{\text {slow }}$ and $\mathrm{D}_{\text {fast }}$ parameters were computed using a Bayesian-probability based fit. The histology analysis included the steatosis, activity, and fibrosis (SAF) score, classifying non-NAFLD, simple steatosis, or NASH. Fibrosis was scored according to the NASH Clinical Research Network (CRN) criteria. According to the SAF-score, NASH was present in 22 of the 37 participants. According to the NASH-CRN criteria, two individuals exhibited no fibrosis (F0), seven patients had $\mathrm{F} 1$ fibrosis, 20 patients had F2 fibrosis, seven patients had F3 fibrosis, and one had cirrhosis (F4). MRE data were not available for two individuals (one F2 case and one F4 case) due to technical issues with the MRE hardware. One IVIM scan (a F2 case) was not included in the analysis due to low image signal-to-noise ratio.

As expected, the results of Troelstra et al. show, the diagnostic performance of MRI based methods generally outperformed ultrasonographic method (11). For liver steatosis evaluation, ROC (receiver operating characteristic) analysis showed similar performance for all three MR methods (MR spectroscopy, magnitude-based MRI proton density fat fraction, and three-point Dixon). Fibrosis grade correlated significantly with disease activity score $\left(r_{\text {spearman }}=0.65\right)$. Steatosis grade did not correlate with either disease activity grade or fibrosis stage.

MRE G' showed a significantly positive correlation with activity grade $\left(r_{\text {spearman }}=0.55\right)$, and comparison of stiffness results for individual activity grades showed a significant difference in medians between the groups. For both PF $\left(r_{\text {spearman }}=0.37\right)$ and $\mathrm{D}_{\text {slow }}\left(r_{\text {spearman }}=0.40\right)$, a significant negative correlation with histological activity grade was noted. $\operatorname{MRE}\left(r_{\text {spearman }}=0.55\right), \mathrm{PF}\left(r_{\text {spearman }}=0.35\right), \mathrm{D}_{\text {slow }}\left(r_{\text {spearman }}=0.37\right)$ correlated significantly with ballooning (11).

There was a significant positive association between MRE G' and fibrosis stage $\left(r_{\text {spearman }}=0.55\right)$, and a significant difference between MRE G' and fibrosis stages. For distinguishing individuals with no/mild (F0-F2) vs. advanced fibrosis stages (F3-F4), the AUROC (area under receiver operating characteristic curve) for MRE G' was 0.92 [95\% confidence interval (CI), $0.83-1$ ], with a sensitivity of $100 \%$ and a specificity of $78.6 \%$. G" showed an AUROC of 0.74 (95\% CI, 0.48-1), with a sensitivity of $71.4 \%$ and specificity of $92.9 \%$. There were significant correlations between $\mathrm{PF}$ $\left(r_{\text {spearman }}=0.53\right)$ and $\mathrm{D}_{\text {slow }}\left(r_{\text {spearman }}=0.46\right)$ and fibrosis grades. Significant difference in medians was noted between PF and fibrosis stages. Among IVIM parameters, PF performed best out of the IVIM parameters for distinguishing between no/mild and advanced fibrosis with an AUROC of 0.88 (95\% CI: $0.72-1$ ), sensitivity of $75.0 \%$ and specificity of $100 \%$. Distinction between no/mild and advanced fibrosis for $\mathrm{D}_{\text {slow }}$ had an AUROC of 0.79 (95\% CI, 0.63-0.95), with a sensitivity of $75.0 \%$ and specificity of $78.6 \%$; while $\mathrm{D}_{\text {fast }}$ had an AUROC of 0.70 (95\% CI, 0.47-0.93), with a sensitivity of $62.5 \%$ and specificity of $75.0 \%$ (11).

For MRE G' and $D_{\text {slow }}$, medians of the NASH group 
were all significantly different compared to the simple steatosis group. For distinguishing individuals with NASH from those with simple steatosis, MRE G' and $\mathrm{D}_{\text {slow }}$ showed an AUROC of 0.79 and 0.73 , respectively. The specificity was highest for MRE at $86.7 \%$, while $\mathrm{D}_{\text {slow }}$ showed the highest sensitivity at $85.7 \%$ (11). The diagnostic performance of MRE for NASH in this study broadly agree with a recent meta-analysis (12).

The limitations of the study of Troelstra et al. include its relatively small sample size, particularly there were only two patients with no fibrosis (F0). Some aspects of correlations may be strengthened if a larger sample was included. Histology was read by a single pathologist, rather than with consensus reading by more than one pathologist (12).

Our overall interpretation of the results of Troelstra et al. is that MRE and IVIM performed at an approximately similar level in fibrosis assessment as well as the differentiation of simple steatosis and NASH, though MRE might have had marginally better performance. In practice when both methods are implemented, their roles would be more complementary.

Along with the global increase in obesity and diabetes mellitus type 2, the prevalence of NAFLD is rising (13). In adults, NAFLD is typically classified into two categories: nonalcoholic steatohepatitis (NASH), a progressive liver disease characterized by a particular pattern of steatosis, inflammatory changes and hepatocellular injury, and simple steatosis which is also called nonalcoholic fatty liver (NAFL). NASH is strongly associated with coronary artery disease and metabolic syndrome (diabetes mellitus type 2, insulin resistance, central obesity, dyslipidemia, and hypertension). NASH can progress to cirrhosis in 21-26\% of the subjects (14). To determine treatment and monitoring plans, distinction between simple steatosis and NASH, and detection and staging of fibrosis are essential. Some degree of fibrosis is often present in livers with NASH (15). Although not a requirement for the NASH diagnosis (16), the presence of fibrosis may help to identify patients with NASH. Fibrosis is a key marker of disease progression in subjects with NASH (15). The stage of fibrosis is the most essential determinant of liver-related progression and mortality, and a key indicator for the development of other comorbidities like type 2 diabetes and cardiovascular disease (17-21). In a longitudinal study of patients with NAFLD, Angulo et al. (22) noted that, fibrosis stage, but no other histologic features of steatohepatitis, was associated independently with long-term overall mortality, liver transplantation, and liver-related events.
The IVIM analyses of our studies and the study of Troelstra $e t a l$. have a number of similarities and differences. Both of us sampled relatively large number of $b$-values, with we aimed to sample $16 b$-values and Troelstra et al. sampled $18 b$-values $(3,11,23)$. Several very low $b$-values (those $<10 \mathrm{~s} / \mathrm{mm}^{2}$ ) were sampled but no $b$-value higher than $700 \mathrm{~s} / \mathrm{mm}^{2}$ was acquired $(2,11)$. Subjects or slices with artifacts were excluded which resulted in one subject was excluded from IVIM analysis in the study of Troelstra et al. (11); and with our results of poor curve fitting were also excluded so that till now we had a higher exclusion rate of approximate $15 \%(5,7-9,24)$. This exclusion rate difference between that of Troelstra $e t$ al. and ours could be due to different exclusion standards were applied. In addition, Troelstra et al. adopted the Bayesian probability analysis. Bayesian analysis allows reasonable estimates at lower signal-to-noise ratio under certain circumstances (25). In this aspect, our laboratory is currently working to reduce our relative high exclusion rate with better image post-processing. While we used METAVIR score for viral hepatitis induced fibrosis and Troelstra et al. used fibrosis score according to the NASH-CRN and all their study participants had hepatic steatosis, our results demonstrated very good results in separating control healthy livers and fibrotic livers with AUROC close to 1 (7-9). Till now, reported data on non-invasive imaging for diagnosis of any NASH fibrosis (F0 vs. F1-4) has been sub-optimal. One recent review suggested MRE had a summary AUC, sensitivity and specificity for diagnosing stage $\geq \mathrm{F} 1$ of 0.87 , $71 \%, 85 \%$, respectively (12). It is possible that additional approaches such as a combination all of all diffusion metrics and removal of $b$-value $=0 \mathrm{~s} / \mathrm{mm}^{2}$ data from IVIM modeling can further improve the results of Troelstra et al. As starting from $b=0$ image, the relationship between liver DWI signal and $b$-value does not follow bi-exponential decay $(2,26)$, in our experience, removal of $b$-value $=0 \mathrm{~s} / \mathrm{mm}^{2}$ data from IVIM modeling greatly improves the diagnostic performance of resulted IVIM parameters $(2,9)$. The fact that slow diffusion measure and fast slow diffusion measures are mutually constrained further increases the necessity of checking all IVIM parameters together (instead of evaluating these parameters individually) $(7,27)$.

Finally, the knowledge of the 'coupling' between slow diffusion measure and fast diffusion measures further highlights the challenges in interpreting IVIM data $(5,27,28)$. According to the currently prevalent IVIM modeling, IVIM parameters are likely affected by biochemical composition of the target tissue as well as diffusion and perfusion of 
this tissue $(29,30)$. Till now, we consider this problem is not easily solvable by a better fitting or a better image post-processing approach. Before improved solutions are available, a practical approach would be that, we standardise the image data acquisition methodology (critically the $b$-value distribution) and image data post-processing/IVIM parameter computing methodology, so to build the database of 'normal' reference distribution of diffusion metrics (the distribution of a reference cluster in 3-dimensional space), then deviation from the reference distribution could be considered an abnormality with fibrosis shown to induce a reduction of all diffusion metrics (23).

\section{Acknowledgments}

Funding: None.

\section{Footnote}

Provenance and Peer Review: This article was commissioned by the editorial office, Annals of Translational Medicine. The article did not undergo external peer review.

Conflicts of Interest: All authors have completed the ICMJE uniform disclosure form (available at https://dx.doi. org/10.21037/atm-21-3442). The authors have no conflicts of interest to declare.

Ethical Statement: The authors are accountable for all aspects of the work in ensuring that questions related to the accuracy or integrity of any part of the work are appropriately investigated and resolved.

Open Access Statement: This is an Open Access article distributed in accordance with the Creative Commons Attribution-NonCommercial-NoDerivs 4.0 International License (CC BY-NC-ND 4.0), which permits the noncommercial replication and distribution of the article with the strict proviso that no changes or edits are made and the original work is properly cited (including links to both the formal publication through the relevant DOI and the license). See: https://creativecommons.org/licenses/by-nc-nd/4.0/.

\section{References}

1. Le Bihan D, Breton E, Lallemand D, et al. MR imaging of intravoxel incoherent motions: application to diffusion and perfusion in neurologic disorders. Radiology
1986;161:401-7.

2. Wáng YXJ. Living tissue intravoxel incoherent motion (IVIM) diffusion $M R$ analysis without $b=0$ image: an example for liver fibrosis evaluation. Quant Imaging Med Surg 2019;9:127-33.

3. Wáng YXJ, Wang X, Wu P, et al. Topics on quantitative liver magnetic resonance imaging. Quant Imaging Med Surg 2019;9:1840-90.

4. Xiao BH, Huang H, Wang LF, et al. Diffusion MRI Derived per Area Vessel Density as a Surrogate Biomarker for Detecting Viral Hepatitis B-Induced Liver Fibrosis: A Proof-of-Concept Study. SLAS Technol 2020;25:474-83.

5. Huang H, Zheng CJ, Wang LF, et al. Age and gender dependence of liver diffusion parameters and the possibility that intravoxel incoherent motion modeling of the perfusion component is constrained by the diffusion component. NMR Biomed 2021;34:e4449.

6. Wáng YXJ. Gender-specific liver aging and magnetic resonance imaging. Quant Imaging Med Surg 2021;11:2893-904.

7. Wáng YXJ, Deng M, Li YT, et al. A Combined Use of Intravoxel Incoherent Motion MRI Parameters Can Differentiate Early-Stage Hepatitis-b Fibrotic Livers from Healthy Livers. SLAS Technol 2018;23:259-68.

8. Huang H, Che-Nordin N, Wang LF, et al. High performance of intravoxel incoherent motion diffusion MRI in detecting viral hepatitis-b induced liver fibrosis. Ann Transl Med 2019;7:39.

9. Li T, Che-Nordin N, Wáng YXJ, et al. Intravoxel incoherent motion derived liver perfusion/diffusion readouts can be reliable biomarker for the detection of viral hepatitis B induced liver fibrosis. Quant Imaging Med Surg 2019;9:371-85.

10. Hu GW, Zheng CJ, Zhong WX, et al. Usefulness of diffusion derived vessel density computed from a simplified IVIM imaging protocol: an experimental study with rat biliary duct blockage induced liver fibrosis. 2021. Available online: https://hal.archives-ouvertes.fr/hal-03221290

11. Troelstra MA, Witjes JJ, van Dijk AM, et al. Assessment of Imaging Modalities Against Liver Biopsy in Nonalcoholic Fatty Liver Disease: The Amsterdam NAFLD-NASH Cohort. J Magn Reson Imaging 2021. [Epub ahead of print]. doi: 10.1002/jmri.27703.

12. Selvaraj EA, Mózes FE, Jayaswal ANA, et al. Diagnostic accuracy of elastography and magnetic resonance imaging in patients with NAFLD: A systematic review and metaanalysis. J Hepatol 2021. [Epub ahead of print]. doi: 10.1016/j.jhep.2021.04.044. 
13. Younossi Z, Tacke F, Arrese M, et al. Global Perspectives on Nonalcoholic Fatty Liver Disease and Nonalcoholic Steatohepatitis. Hepatology 2019;69:2672-82.

14. Matteoni CA, Younossi ZM, Gramlich T, et al. Nonalcoholic fatty liver disease: a spectrum of clinical and pathological severity. Gastroenterology 1999;116:1413-9.

15. Heyens LJM, Busschots D, Koek GH, et al. Liver Fibrosis in Non-alcoholic Fatty Liver Disease: From Liver Biopsy to Non-invasive Biomarkers in Diagnosis and Treatment. Front Med (Lausanne) 2021;8:615978.

16. Brown GT, Kleiner DE. Histopathology of nonalcoholic fatty liver disease and nonalcoholic steatohepatitis. Metabolism 2016;65:1080-6.

17. Dulai PS, Singh S, Patel J, et al. Increased risk of mortality by fibrosis stage in nonalcoholic fatty liver disease: Systematic review and meta-analysis. Hepatology 2017;65:1557-65.

18. Younossi ZM, Stepanova M, Rafiq N, et al. Pathologic criteria for nonalcoholic steatohepatitis: interprotocol agreement and ability to predict liver-related mortality. Hepatology 2011;53:1874-82.

19. Ekstedt M, Hagström H, Nasr P, et al. Fibrosis stage is the strongest predictor for disease-specific mortality in NAFLD after up to 33 years of follow-up. Hepatology 2015;61:1547-54.

20. Sebastiani G, Alshaalan R, Wong P, et al. Prognostic Value of Non-Invasive Fibrosis and Steatosis Tools, Hepatic Venous Pressure Gradient (HVPG) and Histology in Nonalcoholic Steatohepatitis. PLoS One 2015;10:e0128774.

21. Leung JC, Loong TC, Wei JL, et al. Histological severity and clinical outcomes of nonalcoholic fatty liver disease in nonobese patients. Hepatology 2017;65:54-64.

22. Angulo P, Kleiner DE, Dam-Larsen S, et al. Liver Fibrosis, but No Other Histologic Features, Is Associated With Long-term Outcomes of Patients With Nonalcoholic Fatty
Liver Disease. Gastroenterology 2015;149:389-97.e10.

23. Wang YXJ, Huang H, Zheng CJ, et al. Diffusionweighted MRI of the liver: challenges and some solutions for the quantification of apparent diffusion coefficient and intravoxel incoherent motion. Am J Nucl Med Mol Imaging 2021;11:107-42.

24. Chevallier O, Zhou N, He J, et al. Removal of evidential motion-contaminated and poorly fitted image data improves IVIM diffusion MRI parameter scan-rescan reproducibility. Acta Radiol 2018;59:1157-67.

25. Neil JJ, Bretthorst GL. On the use of Bayesian probability theory for analysis of exponential decay data: an example taken from intravoxel incoherent motion experiments. Magn Reson Med 1993;29:642-7.

26. Chevallier O, Wáng YXJ, Guillen K, et al. Evidence of Tri-Exponential Decay for Liver Intravoxel Incoherent Motion MRI: A Review of Published Results and Limitations. Diagnostics (Basel) 2021;11:379.

27. Wáng YXJ. Mutual constraining of slow component and fast component measures: some observations in liver IVIM imaging. Quant Imaging Med Surg 2021;11:2879-87.

28. Wáng YXJ. Observed paradoxical perfusion fraction elevation in steatotic liver: An example of intravoxel incoherent motion modeling of the perfusion component constrained by the diffusion component. NMR Biomed 2021;34:e4488.

29. Xiao BH, Wáng YXJ. Different tissue types display different signal intensities on $b=0$ images and the implications of this for intravoxel incoherent motion analysis: Examples from liver MRI. NMR Biomed 2021;34:e4522.

30. Wáng YXJ. A reduction of perfusion can lead to an artificial elevation of slow diffusion measure: examples in acute brain ischemia MRI intravoxel incoherent motion studies. Ann Transl Med 2021;9:895.
Cite this article as: Wáng YXJ, Xiao BH, Zheng CJ, Li T, Che-Nordin N, Wang W. More promising results of liver intravoxel incoherent motion imaging analysis for the assessment of nonalcoholic steatohepatitis and fibrosis. Ann Transl Med 2021;9(16):1283. doi: 10.21037/atm-21-3442 